\title{
CLOUD HOSTING SERVICES TYPES, FEATURES, ISSUES: A BIG PICTURE
}

\author{
Dharma Raj Ojha ${ }^{1}$, T. Srinivasa Rao ${ }^{2}$ \\ ${ }^{1}$ Student of MCA Department, Sambhram Institute of Technology \\ ${ }^{2}$ HOD of MCA Department, Sambhram Institute of Technology
}

\begin{abstract}
Cloud computing is a type of release based on shared computing resources rather than local server or personal device to handle applications. The cloud computing ("cloud") is provided by computer internet services (server, storage, database, network, software, analysis and more). Cloud facilitating administrations give helping on virtual servers that dismiss their processing properties from the hidden system of physical web server. Generally, customers can aim of their services at any time depending on their needs. This can save cost because they only have to pay and they cannot use it at any time, so they do not have to pay for extra power. You pay for whatever you are using: Since your business requires vacillation, you can change your facilitating bundle as indicated by your necessities, and just pay what you utilize Are there. Decision of OS: You can pick working arrangement of your decision - i.e.Linux or Windows. Adaptability: Finish server arrangement controls by means of a Programming interface or electronic interface. Trickthe two Universes: You can appreciate the advantages of devoted facilitating, yet don't have to tolerate the substantial cost of committed facilitating, on the off chance that you don't have broad necessities.
\end{abstract}

Keywords: Cloud Computing, Cloud Server, Efficiency, Thread Issue.

$* * *$

\section{INTRODUCTION}

Cloud Hosting is an outsourcing provider of supplier's computing and storage resources, which provides its infrastructure services on utility models. Cloud hosting has gained popularity in recent years. If you are looking for your hosting options at any time, you definitely have to face cloud hosting, and you probably have several questions. The cloud concept is a concept that is used in many areas of technology and is now investing in the hosting area. Cloud hosting is a way of establishing a server instead of a clear virtual object, but is expected to say "cloud hosting". In normal server configuration, you will remain limited to the physical extent of your site hosted server. By hosting on the cloud, you are dependent on the same server configuration, which allows you to get from different servers, which translate into a cost-effective hosting solution and are reliably reliable. The simplest definition of hosting in the cloud is the network of virtual servers, which benefit from the following physical servers. The extent of reaching this network depends on the actual requirements of the website and it can be properly expanded or reduced. At the technical level, the resources needed for your website are distributed on multiple remote servers and these resources are used when needed. Your website is not dependent on a server, but the server network, this network of servers is called Cloud.

\subsection{Cloud Hosting Work?}

The easiest way to split hosting into the cloud is to compare it to the same server model. With traditional hosting, you have a server that contains all the files in your website. When users visit your website, these files are provided by the same physical server. Compare this to cloud hosting configurations that can differentiate resources from your site from many different virtual server environments.

The core part of cloud hosting installs is the network of physical servers, which operate on top of virtualized servers. With this configuration, you can get the most endless supply of server resources and measure your site as your needs increase.

\subsection{Cloud Hosting Services}

* Cloud Desktop Hosting Service

Provide global desktop access to your employees at anytime and anywhere. Use the power of hosted virtual desktops to distribute desktop, data and desktop interface applications to any user on the Internet.Cloud desktop hosting services your team works anywhere from your display and gives access to commercial apps like your desk.

With Cloud Desktop Hosting Services from LevelCloud, you can:

- Increase efficiency - Give work area access to end clients around the world, with a similar client encounter, they were in the workplace.

- Escapes of product support- Access your Cloud Work area through your standard internet browser, without the need to introduce or deal with extra programming. 
- Work from anyplace - With electronic worldwide reach on your work area and applications, you can appreciate a similar client encounter on any gadget.

- A use application you cherish - Your cloud work area is introduced with your preferred working arrangement, and you can include fundamental organization particular applications.

\section{* Cloud Application Hosting Services}

Any small or large companies are more than ever in specialized applications to automate major operations. From simple productivity tools to mission-critical commercial equipment, organizations need constant and reliable access to their applications. Using the leading cloud platform Zen up, Lock cloud app will be distributed over hosting services.

Opt for Cloud Application Hosting Services from Level Cloud and:

- Access your applications from the outside, as well as your internal network

- Scale apps more than the general limits imposed by servers and storage infrastructure Supply and maintenance of supplies, and maintenance of servers, storage, and applications

\section{* Cloud SQL Database Hosting Services}

Cloud SQL Database is one of the most important aspects of the data-driven business scenario. Databases advantage from CMS-based sites and online business sites to the administration ventures, to deliver information and data to all successful companies in real-time. Database Contribution (DOS) is unique in the form of the service of Level wood, which offers simple, secure and reliable database management and administration services for each company.Level Cloud's SQL database facilitating is intended to give simplifying and creativity class database helping administrations. This enables you to pick and alter database resources as indicated by your present needs and future necessities, and measure your database in several servers from enormous capacity pools and a huge gathering pool.

Other benefits of Cloud SQL Database Hosting Services from Level Cloud include:

- Unrivaled security - Military review encryption systems to ensure your projectile surge database.

- Web-based administration - Deal with your database wherever you are, as you are on the website.

- Cost reserve funds - Level Cloud level rate choice enables you to lessen costs.

- Expert support - Our insight group is accessible day in and day out each day of the year.

\section{* Cloud Hosted Email Solutions}

The modern organization now depends on your email service, if your email is standing, then it is your company. The absurd availability of email service stops productivity continuously. If you do not play your company's email ball, it's time to do something about it.LevelCloud Enterprise-Class Cloud Host provides email solutions within the range of all sizes. With an auto-service demand on global encryption, collection, virus filtering, scalable storage and global availability, email solutions provided on Levelwood offer a lot. We offer email services to customers in law, service, finance and health care etc.

With Cloud Hosted Email Solutions from LevelCloud, you enjoy:

- 24/7 availability - Constant access to your email anywhere, anywhere

- Scalable storage - Never delete emails with flexible and expandable options

- Native security - Rock-solid security features to protect your communications through email

- Flexible access - Use any Internet enabled device and bring your email with you

- Cost savings - Find out what you are spending with our low cost monthly billing model

\section{* Cloud File Server Hosting Services}

Modern companies develop data. However, data stored only through internal network or file servers can be accessed through internal networks, but actual employees want continuous access to their data from anywhere. They want to reach continuous, trustworthy and safe, often not guaranteed by internal measures. With Cloud Client server hosting services from Level Cloud, you can enjoy some more. They allow organizations to create, manage and manage file hosting on a cloud basis and share services for better performance. Our cloud file server hosting services are specially designed to help companies compensate for storage and security, security and file access issues to ensure that your company is always within the scope of your important data.

Cloud File Server Hosting Services from Level Cloud offer:

- Global accessibility - Access all your files quickly

- Device compatibility - Use any phone, tablet or laptop to access all your data

- Secured sharing - Encryption provides reliable data support

- Reliable uptime - Power backup, internet and fuel your confidence

- Cost efficiency - Scalability and better productivity can help you save money

- Legally compliant - To ensure that it adheres to industry and legal provisions

\subsection{Security Issues}

Cloud Computing Between a growing business setting, security professionals are not surprised that host of cloudbased servers are looking for security mechanisms for 
security. This effort is increasingly looking at the techniques used to protect data centers such as firewalls, antivirus and infiltration detection systems. This is a logical way; they need to keep cloud-based resources safe as local resources. However, the risk of traditional security technology was designed to focus solely on local safety, thereby limiting their effectiveness while trying to secure resources on cloud basis. Fortunately, Cloud Computing has many new types of security products based on the cloud provided in the service offered to reduce the risks.

\subsection{Solution}

Encryption: Cloud computing models want many clients to reduce IT costs by using shared computing platforms. This means that the company's most confidential data can be kept separate from other customers through the main index field in the database. Cloud-based encryption can ensure that this information is kept private. These services can be easily integrated with major cloud hosting providers and orders such as FIPS-140-2, PCI DSS and HIPAA-HITECH can be followed.

Configuration and Vulnerability Management: It's easy to lose the number of virtual servers created in the cloud environment. The advantage of using cloud computing is that these virtual servers can be built quickly to meet business needs. However, many cloud service providers have created servers in the environment; tracking, configuration and robustness of each virtual server quickly become an important challenge for most businesses.

\section{CONCLUSION}

It provides complete flexibility to add more systems (servers) to handle additional loads (expected or unexpected). Therefore, in reality many models of no downtime can be measured. Cloud servers provide better security and stability. In large part, this is due to a different environment where the cloud server installation can be implemented. Second, if one of the physical servers compromises, then you can rely on any other server that works correctly. The easiest way to split hosting into the cloud is to compare it to the same server model. With traditional hosting, you have a server that contains all the files in your website. When users visit your web site, these files are provided by the same physical server.Compare this to cloud hosting configurations that can differentiate resources from your site from many different virtual server environments. The core part of cloud hosting installs is the network of physical servers, which operate on top of virtualized servers. With this configuration, you can get the most endless supply of server resources and measure your site as your needs increase.

\section{REFERENCES}

[1] Kevin Wood-Web hosting tips, February 27, 2018

[2] Om Thoke-Understanding cloud hosting, march 06, 2017
[3] Joseph Granneman-Cloud security as service for secure cloud-based server hosting

\section{Online}

[1] https://www.lifewire.com/understanding-cloudhosting-3473590

[2] http://www.cetrom.net/cloud-services/cloud-hostedemail-solution/

[3] https://www.hostgator.com/blog/what-is-cloudhosting/

[4] https://www.levelcloud.net/ 